\title{
BARGAIN STRATEGIES FOR AGENT AUTOMATED NEGOTIATION IN AN E-BUSINESS ENVIRONMENT
}

\author{
SERBAN RADU AND ADINA MAGDA FLOREA*
}

\begin{abstract}
An automated negotiation environment, in which agents employ different bargaining strategies is described. During negotiation, as more information is exchanged in the negotiation rounds, the agents can change the preferences for certain attributes of the negotiation object. The multi-agent system is developed for a real estate agency business model and several use cases scenarios, using intelligent software agents, are implemented.
\end{abstract}

Key words: automated negotiation, multi-agent system, negotiation strategy

AMS subject classifications. 68T42, 68T05, 68T35

1. Introduction. Negotiation is an important issue in business environments. Automated negotiation is a process, in which software agents communicate between them, in order to reach a mutually acceptable agreement [1]. An intelligent agent should be able to negotiate with other agents, which have different negotiation behaviors [2]. Best outcome may be obtained if the agent is able to adjust its strategy, predict or guess the strategy of the other agent [3], or choose an adequate strategy, according to the negotiating partner. Different approaches have been proposed, including machine learning approaches, which can be used to change the agent strategy during negotiation, in order to obtain better results and increased payoffs [4].

In our previous works regarding the automated negotiation process, we have proposed bargaining strategies that are based on agent profiles, which can describe statically or can develop dynamically the agent preferences for certain attributes of the negotiation object [5,6]. Using these profiles, agents obtain better results than in the case when fixed negotiation strategies are employed.

This paper extends our previous work and proposes an agent model, in which the agents apply the negotiation strategy best suited to them, according to the negotiation situation. A multi-agent environment for automated negotiation is designed, offering services for a real estate business model. In the multi-agent system, there are buyer and seller agents and also a facilitator, which is used when a new agent enters into the system, for registering its services. The agents are designed according to the BDI (Belief-Desire-Intention) model [7]. Each agent has a set of goals, selected from the set of desires.

Several use cases scenarios evaluate the negotiation performances. The bargaining takes place in several rounds, before an agreement or a rejection is concluded. In each negotiation round, based on the values and preferences specified by the buyer for the multiple attributes of the negotiation object, the seller agent makes the best possible offer. The agents use linear and non-linear negotiation strategies, which help them in time to increase the gain.

The paper is organized as follows: Section 2 presents the negotiation environment, that is our proposed framework for the negotiation system in an open environment. The agent behavior and how each agent acts during the negotiation process is described in Section 3. In Section 4 is applied the developed negotiation environment for the business model of a real estate agency. The related work is presented in Section 5, while Section 6 discusses conclusions and future work.

2. The Negotiation Environment. The negotiation system consists of cognitive agents, which use a set of negotiation primitives, implemented in the Iterated Contract Net protocol, cf. Fig. 2.1 [8].

The environment is open and the agents are able to enter and leave the environment at any time. The multi-agent system models a heuristic negotiation, in which the agent computes the gain, with respect to its private value for the negotiation object. The agent uses a set of rules to choose the negotiation strategy and to follow that strategy. Also, there is a deadline for the number of negotiation rounds. In a buying negotiation, an agent will look for a lower value than its private value, while in a selling negotiation, its main goal is to obtain more than the item's private value.

\footnotetext{
*Computer Science Department, University Politehnica of Bucharest, Bucharest, Romania (serban.radu, adina.florea@cs.pub.ro).
} 


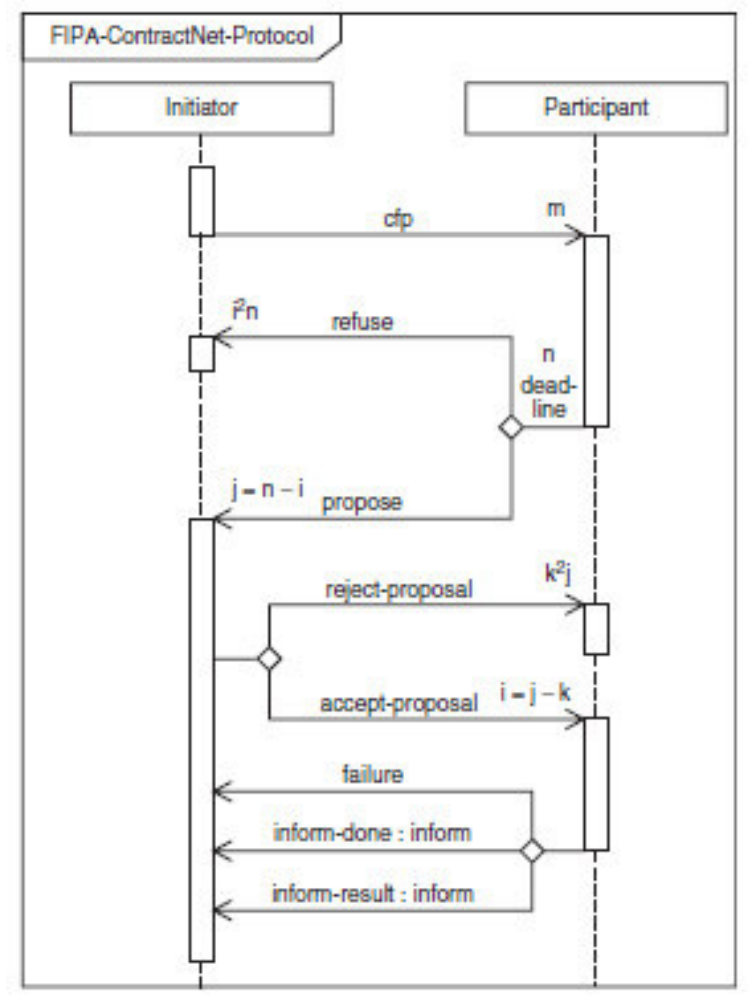

FIG. 2.1. FIPA-Contract Net protocol. [8]

The agent behavior is mainly motivated by the gain, but also, depending on a specific context, by the desire to achieve cooperation with other agents. For example, as a general rule, the agent will not accept a price lower than its private value. However, when the agent wants to cooperate, it can accept a lower price. The agent behavior is set up by the negotiation strategy.

The negotiation environment has the following three features [9]:

a) When acquiring multiple goods, a buyer agent only knows the private value available for the set of items, that is the highest price the agent can pay for all the goods, rather than the private value of each separate item;

b) Agents can decommit from tentative agreements at the cost of paying a penalty. Decommitment allows agents to profitably accommodate new negotiations. If these negotiations make some existing contracts less profitable or infeasible for an agent, that agent can decommit from those contracts;

c) Negotiation agents are assumed to have incomplete information about other agents, for example, a buyer agent knows the distribution of the private value of a seller agent and the number of trading competitors. However, an agents negotiation status (the set of proposals it has received) and negotiation strategy are its private information. During negotiation, the agents can quit negotiation at any time, even without notifying their trading partners. When an agent wants to buy multiple goods or services, it concurrently negotiates with sellers to reach agreements for all the items.

In order to evaluate the performance of negotiation agents, a simulation environment consisting of agents negotiating in the business model of a real estate agency, are modeled and implemented in this approach.

In the experiments, agents are using different negotiation strategies, deadlines, and objects to buy or sell. A number of performance measures, such as utility, gain, number of successful negotiations, learning capabilities, are determined.

A negotiation object represents the range of features and issues over which agreements must be reached. In case of single-issue negotiation, the object of negotiation may be either a unique item, for example a good that the agent $A_{1}$ wants from $A_{2}$ or a service that agent $A_{1}$ offers to $A_{2}$. In case of multi-issue negotiation, the 
negotiation object is replaced by a negotiation package, composed of a set of negotiation objects.

Each agent has two prices, the minimum price and the maximum price, between which it accepts offers. The buyer computes its gain as the difference between the maximum price it is willing to pay and the price of the current offer. The seller computes its gain as the difference between the price of the current offer and the minimum price it is willing to accept.

An agent is focused on a set of useful strategies. These strategies can be predefined or, alternately, can be learned in time, such that to choose the best strategy for negotiating with a certain agent. There are a lot of factors which can influence the result of a negotiation strategy. These factors refer to the strategies of other agents, their constraints and preferences, and other characteristics of the negotiated issues. The strategies are rule-based and are reusable for different negotiation problems, with different agents.

Agents use linear and non-linear strategies, with the non-linear strategies divided in: conceder strategy, if the agent is willing to concede a lot in the first rounds of negotiation, and boulware strategy, if the agent is willing to concede considerably only when the time deadline is close [10].

A seller agent starts by making an offer that represents a high price, and over time, as it makes concessions, the price is decreased. The difference between the non-linear strategies lies in the way in which the price comes down. In the conceder strategy, the agent makes all its concessions early and then doesn't concede much, as the negotiation evolves and the deadline approaches. In the boulware strategy, the agent doesn't initially change the price so much at each time step. But, as the deadline for negotiation approaches, the size of its concessions increases by larger amounts. During the bargaining process, these negotiation strategies are used in our environment, both by buyers and sellers.

Different negotiation scenarios are tested, in an open and adaptive multi-agent system. The framework is implemented using Java, Jade, Jess, and XML. The system has the possibility to add or remove agents during run-time, showing that it is an open environment. Jade is used as an infrastructure for creating the multi-agent system and Jess is a mechanism for providing the inference engine for the agents that are negotiating. The Jess engine represents the agent inference engine, which stores the knowledge base of the business domain and also contains the negotiation strategies of the agent, the facts and the concepts of bargaining. Also, Jess rules describe the way in which the price is computed during negotiation, with respect to the negotiation strategy of the agent.

During negotiation, an agent wants to maximize its gain, by fulfilling its goals. Also, negotiation criteria refer to the cooperation profile the agent has developed to describe the interaction history with other agents in the system. The cooperation profile is considered as a part of the agent belief about other agents in the system.

3. Agent Behavior. The agents have different reasoning capabilities, used to conduct successful negotiation and to reach their goals. Each agent learns in time which strategy gives the maximum utility [11]. For doing this, each agent has an associated utility values matrix, which helps the agent to choose the strategy which maximizes its utility.

Any agent from the system uses a set of behavior rules, which define how the agent fulfills the goals, and a set of strategy rules, which guides the negotiation process.

The negotiation strategy of an agent may show how much and how quickly the prices are decreased and if the price is lower than the private value of the agent. An agent can use a tradeoff in negotiation. In the case of cooperative agents, an agent can gain more once, and then can sell cheaper. This is a kind of global evaluation on previous deals.

The negotiation strategy is implemented in the form of rules, each agent keeping a history of its interactions. If in a given situation, several rules are eligible, then the negotiation strategy decides which rule, from the conflict set, to be applied. A possible approach to solve the conflicts is to assign priorities between rules. The solution is to apply the rule with the highest priority. The priority of the rules is dynamically modified, according to the negotiation situation.

The agents in the system use different strategies, for instance, at each negotiation step, the price is decreased by 1 , or is decreased by 3 . Another strategy rule tells what happens when the price increases with $10 \%$ above the private value, if the offer is instantly accepted or not.

In our research, the cooperation profile describes the preferences about the agents with which an agent wants to cooperate. This profile is implemented as a dynamic structure. For each agent with which a negotiation is 
performed, the knowledge regarding the negotiation result is stored in the cooperation profile. This is updated during the negotiation process, at the end of each negotiation.

The classification of the partner agent represents the current agent belief about the cooperation potential of the partner. The partners are classified into six cooperation classes: highly cooperative, very cooperative, cooperative, slightly cooperative, non-cooperative, and unknown [5]. While more negotiations take place, the cooperation class associated to the partner agent can be changed. According to the negotiation history with a certain agent and depending on the cooperation class of the partner, the agent learns in time which negotiation strategy offers the maximum gain.

The characterization of the cooperation potential of a partner agent is done by classifying the partners into cooperation classes, which divides the cooperation ability of the partner into six classes. The classification is done using the C4.5 learning algorithm, in which a decision tree is a classifier for the cooperation degree, expressed as a recursive partition of the instance space. In the decision tree, the class is represented by the partner classification field. During the negotiation rounds, the C4.5 algorithm can classify the partner agent into another cooperation class, if the values of the attributes used in the algorithm are changed.

In the statistics file, associated to the multi-agent system, the following information is collected:

a) Negotiation Statistics - for each agent, the system collects the total gain, the number of negotiations, and the total number of negotiation rounds;

b) Cooperation Statistics - for each agent and partner cooperation class, the system collects the number of negotiation rounds, the number of negotiations, the total gain, and the list of agents included in that cooperation class;

c) Supply-Demand Statistics - supply is computed as the sum of quantities of all seller products and demand is computed as the sum of quantities of all buyer products. Their ratio is computed by dividing the demand to the supply.

In the implemented system, developed in this research, there is a base agent class, extended by buyer and seller agents, which contains common functions used by all agents. The main features of this class are described in the following steps:

1. Read the negotiation object;

2. Check if the current agent has objects with the desired attributes;

3. If there are no objects in the stock then REJECT-PROPOSAL;

4. Check the message type;

5. If message=CALL-FOR-PROPOSAL (only sellers receive CFP) then get all objects of that type;

5.1. If there are less objects than required then REJECT-PROPOSAL;

5.2. If there are offers then send them;

6. If message $=$ INFORM (only buyers receive INFORM) then

6.1. If multiple offers are received then find which one is the best;

else check if the attributes' values match the request;

7. If message=PROPOSE then process offer;

8. If message $=$ AGREE then

8.1. Add information to statistics file and compute gain;

8.2. Remove object from stock;

8.3. Collect statistics when negotiation ends;

9. If message=REJECT-PROPOSAL then end negotiation with reject.

Each buyer agent does the following steps:

1. Every 5 seconds the buyer agent sends to all sellers REQUESTS for the objects of interest;

2. Get the agents which sell what the agent needs to buy.

Each seller agent performs the following steps, when processing an ACCEPT offer:

1. Remove the object from the stock;

2. If agreement on price is set then sends ACCEPT message to the buyer agent;

3. Add the negotiation results to statistics and compute the gain;

4. Remove the current bid from the list of open bids;

5. Reject the offers of other agents interested in this object; 
TABLE 4.1

The Buyer Agents Requirements and the Seller Offer in the Three-to-One Automated Negotiation Scenario, with Two House Types.

\begin{tabular}{|c|c|c|c|}
\hline Agent & House Type & Quantity & Price \\
\hline$B_{1}, B_{2}, B_{3}$ & 2 Rooms & 5 & $40000-60000$ \\
\hline$B_{1}, B_{2}, B_{3}$ & 3 Rooms & 5 & $80000-100000$ \\
\hline$S_{1}$ & 2 Rooms & 20 & $45000-70000$ \\
\hline$S_{1}$ & 3 Rooms & 20 & $85000-110000$ \\
\hline
\end{tabular}

\section{Add to statistics file the result of the failed negotiations.}

4. Real Estate Agency Automated Negotiation Business Model. An automated negotiation environment between real estate agencies and real estate developers is realized. The houses to be sold have different attributes, expressed in the XML configuration file, associated to this business model. The negotiation is done based on price, but there are also other attributes in the configuration file, such as the number of rooms, rooms' dimensions, quality of finishes, location of the house.

The negotiation process starts when the buyer agent wants to buy a house. A request for the house is sent to all sellers. These agents respond to the request with their configuration for the house. After receiving the initial offers, the buyer evaluates and compares different offers. When an offer is not satisfactory, the buyer agent makes a counterproposal to the corresponding seller agent. This has a set of strategies that configure its constraints. The particular strategy used in a certain moment of negotiation depends on the market dynamics and can be changed from the graphical interface associated with each agent. When the strategy is changed, the suitable Jess rules are applied by the agent.

The buyer agent from the real estate agency scenario has several criteria, represented by attributes, each having a certain priority for the user. These characteristics are encoded in the XML configuration file, associated to the business model. The content of the configuration file is read into the application using the $\mathbf{S A X}$ parser.

The rules, upon which the negotiation is performed, are defined in Jess. There are different rules defined for each type of the communication primitive. The higher priority is associated to the ACCEPT rules, the medium priority to the REJECT rules, and the lower priority to the PROPOSE rules.

The negotiation is ended when one of the agents sends an ACCEPT message, or when the time expires, and a REJECT message is concluded. There are different rules associated to each communication primitive of the agents, and also for each strategy of the agent.

Some examples of Jess rules, upon which the negotiation is performed in the real estate agency business model, are described in what follows:

a) The first ACCEPT rule says that an offer for a quantity $q$ for a certain product, having the price between minPrice and maxPrice, from an unknown or non-cooperative partner, is accepted, if the price is less than $q^{*}($ minPrice + maxPrice $) / 2$;

b) When using the conceder strategy, the buyer increases its previous offer with 800, towards very cooperative or highly cooperative sellers, when the negotiation step is greater than 12;

c) When using the boulware strategy, the seller decreases its previous offer $o$ by 2000, when the negotiation step is greater than 1 and the time elapsed in the negotiation is greater than 4.5 seconds, represented by the global variable boulwareTime2;

4.1. Three-to-One Automated Negotiation Scenario. The following scenario involves a real estate agency. There are three buyers, wanting to buy houses from the real estate agent, each buyer having a different negotiation strategy: $B_{1}$-linear, $B_{2}$-conceder, and $B_{3}$-boulware. The seller $S_{1}$ has a linear strategy. The requirements of the agents are represented in Table 4.1.

Figure 4.1 represents a screen capture of the Jade Sniffer Agent for this scenario, showing the messages exchange between agents.

The next diagrams (cf. Figs. 4.2 and 4.3) are obtained using the data collected in the statistics file, generated after the negotiation is performed between the agents in the platform. When all the buyers finish their purchases, the negotiation information is recorded in the statistics file.

The buyers gain, obtained after negotiation, is represented in Fig. 4.2. 


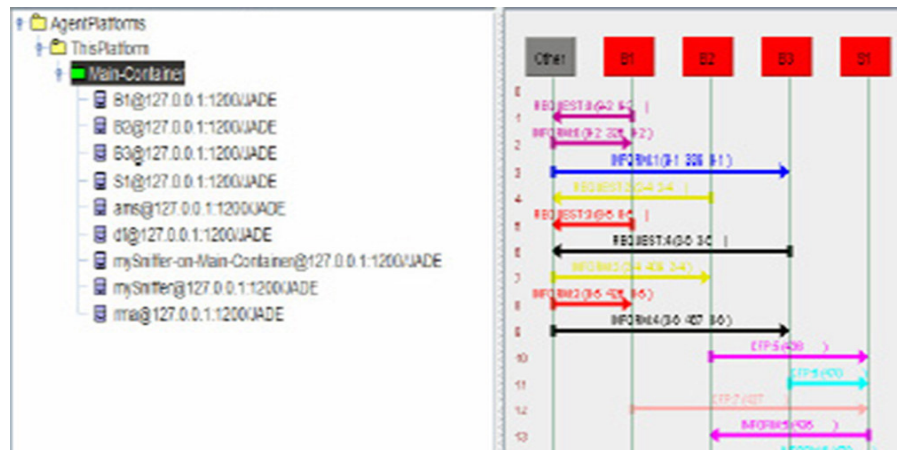

FIG. 4.1. Screen capture showing the messages exchange between three buyers and one seller.

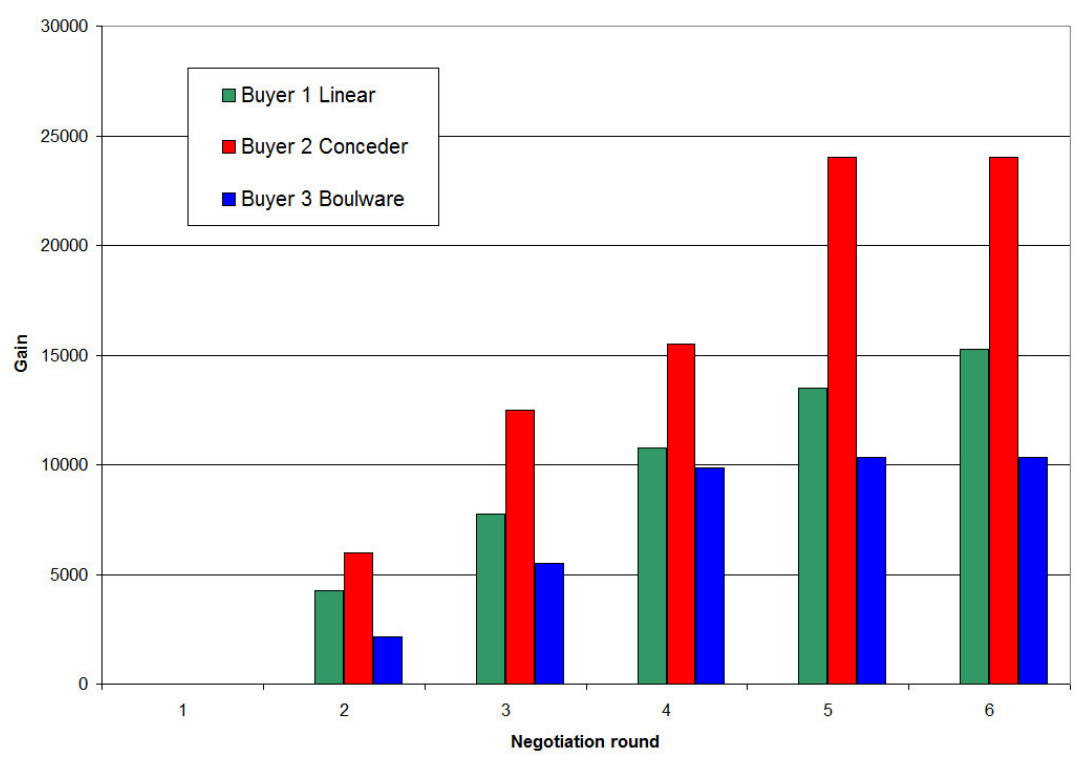

FIG. 4.2. The three buyers gain, each using a different negotiation strategy.

With respect to the gain of the buyers obtained after negotiation, the higher gain is obtained for the buyer using the conceder strategy, the medium gain corresponds to the linear strategy and the smaller gain is for the boulware strategy.

4.2. Two-to-Three Automated Negotiation Scenario. The next scenario involves two buyers and three sellers, having the requirements displayed in Table 4.2. The buyers use a linear strategy, but the sellers are using different strategies: $S_{1}$ has a linear strategy, $S_{2}$ a conceder strategy, and $S_{3}$ a boulware strategy.

Figure 4.3 represents the sellers gain versus the negotiation rounds. Each seller uses a different strategy during negotiation.

Regarding the gain of the sellers obtained after negotiation, the higher gain is obtained for the seller using the boulware strategy, the medium gain corresponds to the linear strategy and the smaller gain is obtained for the conceder strategy.

5. Related Work. There are some different approaches in the automated negotiation domain. A negotiation system is presented in [12], which supports the design of different strategies for agent negotiation, and the evaluation of these strategies in a simulated environment. The designer of a strategy can select from a repository, a negotiation domain and a preference profile for the agent. As compared to this system, in our 
TABLE 4.2

The Buyer Agents Requirements and the Sellers Offer in the Two-to-Three Automated Negotiation Scenario, with Three House Types.

\begin{tabular}{|c|c|c|c|}
\hline Agent & House Type & Quantity & Price \\
\hline$B_{1}$ & 2 Rooms & 5 & $40000-60000$ \\
\hline$B_{1}$ & 3 Rooms & 5 & $80000-100000$ \\
\hline$B_{1}$ & 4 Rooms & 5 & $100000-120000$ \\
\hline$B_{2}$ & 2 Rooms & 5 & $45000-65000$ \\
\hline$B_{2}$ & 3 Rooms & 5 & $85000-105000$ \\
\hline$B_{2}$ & 4 Rooms & 5 & $105000-125000$ \\
\hline$S_{1}$ & 2 Rooms & 10 & $50000-70000$ \\
\hline$S_{2}$ & 3 Rooms & 10 & $90000-110000$ \\
\hline$S_{3}$ & 4 Rooms & 10 & $110000-130000$ \\
\hline
\end{tabular}

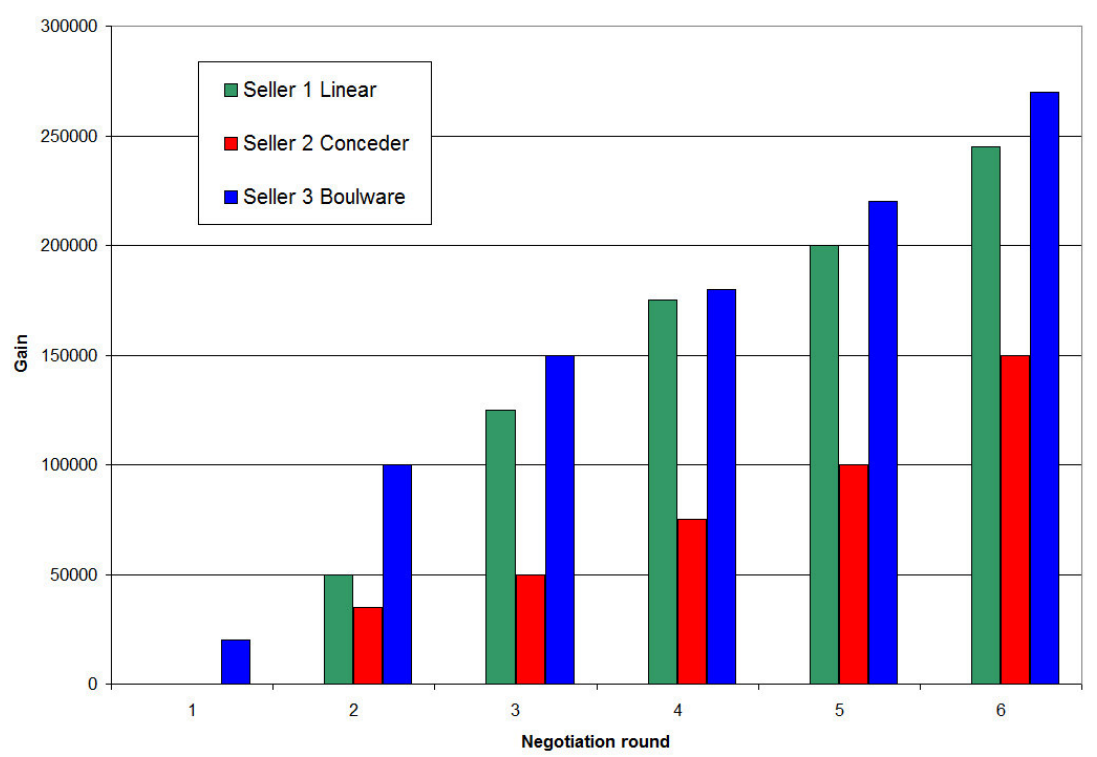

FIG. 4.3. The three sellers gain, each using a different negotiation strategy.

approach, there are several negotiation strategies, which can be changed during negotiation, and the negotiation domain is specified by the agent rules.

A negotiation strategy that describes a method to learn a model of opponent preferences in a single negotiation session is presented in [13]. The negotiation strategy should be efficient, transparent, maximizing the chance of an agreement and should avoid exploitation. In the current paper, we have applied different negotiation strategies, in order to improve in time the behavior of the agents.

A model of iterative reasoning process is developed in [3], by widening the notion of a level in a hierarchy, from one single strategy to a distribution over strategies, leading to a more general framework of multi-agent decision making. Our approach combines different strategies, in order to find the best possible outcome.

The impact of the negotiation environment on the performance of several intra-team strategies is studied in [14]. An agent-based negotiation team is a group of agents that joins together, because they share common interests in the negotiation. In our approach, the agents are self-interested and are using a set of strategies, towards achieving their goals, trying to obtain the maximum gain.

An agent, which learns the general pattern of behavior, based on all of the interactions in which it participates is developed in [15]. A generic approach, which may help the agent compete against unknown opponents in different environments is proposed. In our research, the agents' beliefs about the other agents in the system are combined with the possibility to represent and modify dynamically the negotiation strategy. 
The decision to accept an offer is presented as a sequential decision problem in [16], by modeling the bids received as a stochastic process. This choice is useful in the context of negotiation with incomplete information, where the future behavior of the opponent is uncertain. In our approach, the agents use the Iterated Contract Net protocol, which has the advantage that it can simulate a real-world scenario, with many buyers and sellers having parallel negotiations.

A multi-issue negotiation that runs under real-time constraints and in which the negotiating agents have no prior knowledge about their opponents' preferences and strategies is described in [17]. A negotiation strategy, which employs sparse pseudo-input gaussian processes to model efficiently the behavior of the negotiating opponents, is used. In our approach, the strategy is represented in the form of rules, with their attached preference coefficients.

An agent, which estimates the opponent's strategies based on the past negotiation sessions, is presented in [18]. The agent tries to compromise to the estimated maximum utility of the opponent by the end of the negotiation. In our system, the agents' behavior can change during negotiation, according to previous interactions with other agents. Changing behavior may refer to either the use of different negotiation strategies or to concessions made for other agents, with which they have successfully negotiated in the past.

Modeling the decision-making process, through the design of a goal directed negotiation model, based on the BDI agent theory, is developed in [19]. A prototype of the model is built and applied to an aircraft purchase negotiation process. Also, in our system, the agents are designed according to the BDI model. Automated negotiation agents are developed, having adaptive negotiation strategies. The model is implemented and the agent behavior is tested on different settings, for a real estate agency business model.

6. Conclusions and Future Work. A multi-agent system for automated negotiation, involving a real estate agency business model, was presented. The gain obtained by agents during negotiation is computed after each negotiation round and is graphically represented.

The negotiation strategy of the agents can be changed in time, as more negotiations are taking place. The agents have a set of useful negotiation strategies, from which they can choose the best one. The three possible negotiation strategies: linear, conceder, and boulware, can be dynamically changed, during run-time, for each negotiating agent, being either buyer or seller, using the graphical interface of each agent. The combination of strategies for buyer and seller agents gives different gains for the agents.

The experiments demonstrate distinct behavior and gain for the agents employing different strategies. The strategy used by agents is dependent on the number of buyers and sellers in the virtual market. Also, the tests performed showed the improvement of the agents performance in time, with respect to their negotiation abilities.

Because the agents preferences are based on their goals, changes of their needs influence the preferences during negotiation. When new information is available, the agents can update their preferences regarding the negotiation results. The communication primitives and the framework can express complex negotiation dialogues, in which agents can change their preferences in time.

Heuristic negotiation strategies used in this article are based on the exchange of proposals. In the case the answer received from the partner agent is a counterproposal, the argumentation-based negotiation extends the negotiation protocol with the possibility to exchange arguments. This information gives explicitly the opinion of the agent making the argument.

Future work will investigate the arguments used by agents for improving the negotiation outcomes. Also, future work will be directed towards the implementation of more complex agent strategies and knowledge sharing ability between agents.

Acknowledgments. This work was supported by the project ERRIC No. 264207, FP7-REGPOT-2010-1.

\section{REFERENCES}

[1] N. R. Jennings, P. Faratin, A. R. Lomuscio, S. Parsons, M. J. Wooldridge, And C. Sierra, Automated Negotiation: Prospects, Methods and Challenges, International Journal of Group Decision and Negotiation, 10, 2 (2001), pp. $199-215$.

[2] S. S. Fatima, M. J. Wooldridge, and N. R. Jennings, On Optimal Agendas for Multi-Issue Negotiation, Proceedings of the 12-th International Workshop on Agent-Mediated Electronic Commerce, 2010, pp. 155-168. 
[3] M. Wunder, M. Kaisers, J. R. Yaros, And M. Littman, Using Iterated Reasoning to Predict Opponent Strategies, Proceedings of the 10-th International Conference on Autonomous Agents and Multiagent Systems, 2 (2011), pp. 593-600.

[4] S. Manju And M. Punithavalli, An Analysis of Q-Learning Algorithms with Strategies of Reward Functions, International Journal on Computer Science and Engineering, 3, 2 (2011), pp. 814-820.

[5] S. Radu, E. Kalisz, And A. M. Florea, A Model of Automated Negotiation Based on Agents Profiles, Scalable Computing: Practice and Experience Journal, 14, 1 (2013), pp. 47-55.

[6] S. Radu, E. Kalisz, and A. M. Florea, Automatic Negotiation with Profiles and Clustering of Agents, International Journal of Intelligence Science, 3, 2 (2013), pp. 69-76.

[7] M. Georgeff, B. Pell, M. Pollack, M. Tambe, and M. J. Wooldridge, The Belief-Desire-Intention Model of Agency, Intelligent Agents V: Agents Theories, Architectures and Languages, Lecture Notes in Computer Science, 155 (1999), pp. $1-10$.

[8] F. Bellifemine, G. Caire, and D. Greenwood, Developing Multi-Agent Systems with JADE, John Wiley \& Sons Ltd, UK, 2007.

[9] B. An, V. Lesser, And K. M. Sim, Strategic Agents for Multi-Resource Negotiation, Autonomous Agents and Multi-Agent Systems, 23, 1 (2011), pp. 114-153.

[10] M. Wooldridge, An Introduction to MultiAgent Systems, Second ed., John Wiley \& Sons Ltd, UK, 2009.

[11] S. Radu And V. Lungu, An Adaptive Multi-Agent Model for Automated Negotiation, Proceedings of the 19-th International Conference on Control Systems and Computer Science, 1 (2013), pp. 167-174.

[12] R. Lin, S. Kraus, J. Wilkenfeld, And J. BARry, Negotiation with Bounded Rational Agents in Environments with Incomplete Information using an Automated Agent, Journal of Artificial Intelligence, 172 (2008), pp. 823-851.

[13] K. Hindriks, C. M. Jonker, And D. Tykhonov, The Benefits of Opponent Models in Negotiation, Proceedings of the 2009 IEEE/WIC/ACM International Joint Conference on Web Intelligence and Intelligent Agent Technology, 2 (2009), pp. $439-444$.

[14] V. Sanchez-Anguix, V. Julian, V. Botti, And A. Garcia-Fornes, Studying the Impact of Negotiation Environments on Negotiation Teams' Performance, Information Sciences, 219 (2013), pp. 17-40.

[15] R. Azoulay, R. Katz, and S. Kraus, Efficient Bidding Strategies for Cliff-Edge Problems, Journal of Autonomous Agents and Multi-Agent Systems, 28 (2014), pp. 290-336.

[16] T. BaArslag And K. V. Hindriks, Accepting Optimally in Automated Negotiation with Incomplete Information, Proceedings of the 2013 International Conference on Autonomous Agents and Multi-Agent Systems, 2013, pp. 715-722.

[17] S. Chen, H. B. Ammar, K. Tuyls, and G. Weiss Optimizing Complex Automated Negotiation using Sparse Pseudo-Input Gaussian Processes, Proceedings of the 2013 International Conference on Autonomous Agents and Multi-Agent Systems, 2013, pp. 707-714.

[18] K. Fujita Automated Strategy Adaptation for Multi-Times Bilateral Closed Negotiations, Proceedings of the 2014 International Conference on Autonomous Agents and Multi-Agent Systems, 2014, pp. 1509-1510.

[19] M. CAo Goal Deliberated Agent Architecture for Multi-Strategy Selection in Automated Negotiation, Proceedings of the 14-th Annual International Conference on Electronic Commerce, 2012, pp. 159-160.

Edited by: Florin Fortiş

Received: Mar 1, 2014

Accepted: Jun 24, 2014 